\title{
The chaotic system of simplify-memristor simulator in jerk circuit
}

\author{
Junjie Wen, Miao Miao*, Huizhen Yan \\ \{miaomiao@dlpu.com\} \\ School of Information Science and Engineering, Dalian polytechnic University, Dalian, 116034, China

\begin{abstract}
The paper puts forward a simplified jerk circuit simulator, based on a simplified have resistance device emulator jerk circuit with unstable saddle points. According to the circuit structure, circuit of the dimensionless model is set up, in the simulation At the same time, many basic dynamic properties, such as stability and equilibrium, are analyzed. The dynamic characteristics in this system are analyzed by the commonly used dynamic analysis methods such as Lyapunov index and bifurcation diagram, and several special dynamic phenomena are observed. For instance: chaos, quasi-periodic, periodic, and some complex behavior. The research results, which will offer experimental basis and theoretical for the application of the chaotic system pseudorandom sequence in the field of public information security, for instance, digital communication security.
\end{abstract}

Keywords: Jerk circuit, Simplified-memristor, Chaos

\section{Introduction}

Over the past few years, this inherent nonlinearity of memristors [1] has often been used to design new nonlinear chaotic circuit and system is formed by using memristor to replace the non-linear or linear elements in the existing chaotic circuit system, resulting in a rich variety of non-linear dynamic behaviors [2-15] including quasi-period, period, chaos. Because so far, it has the required structure relationship of today also can't buy in the market, so many have been proposed by the ready-made discrete component implementation of today the emulator, they mainly composed of operational amplifier and analog circuit based on multiplier today with different nonlinear device simulator and related to the second-order and first-order filter network have resistance model. The memristor simulators realized by these equivalent circuits are suitable for the research of hardware experiments. In addition, the jerk system [16-18] is a 
simple form in the system's algebraic structure, achieving compact circuits with fewer circuit elements. For this reason, this paper introduces a simplified memristor emulator into the existing jerk circuit. Six discrete elements constitute a simplified memristor [19]: integrator $U_{1}$, two resistors $R$, a capacitor $C$, an analog multiplier $\mathrm{M}_{1}$ and a resistor $R_{\mathrm{a}}$. Integrating capacitance $C$ and resistor $R$ are used in parallel in order to avoid DC voltage integration drift. Compared with the proposed memristor, the simplified memristor has the advantages of simple mathematical model and easy circuit system realization.

The paper is laid out as follows. In the second part, a simplified memristor simulator model is introduced, and the shrinkage hysteresis loops are validated by calculation and experiment. We put the dynamic analysis of chaotic system in the third part, and this conclusion of this paper is obtained in the last chapter.

\section{Simplified memristor simulator}

For convenience study the chaos dynamics of jerk circuits based on memristor simulators, a simplified memristor [19] simulator is proposed based on the references. The simplified memristor [19] simulator consists of six discrete elements: an integrator $U_{1}$, a capacitor $C_{0}$ and two resistors $R_{1}$, a resistor $R_{e}$ and an analog multiplier $A_{1}$. Integrating capacitance $\mathrm{C}$ and resistor $\mathrm{R}$ are used in parallel in order to avoid DC voltage integration drift. Compared with the proposed memristor, the simplified memristor has the advantages of simple mathematical model and easy circuit system realization.

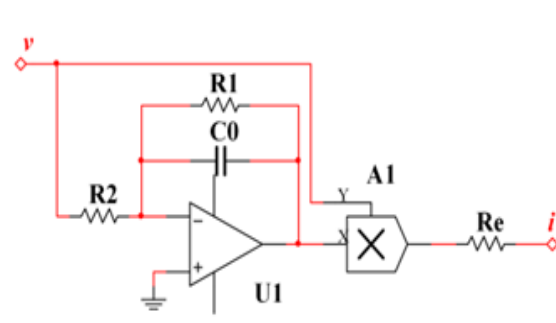

(a)

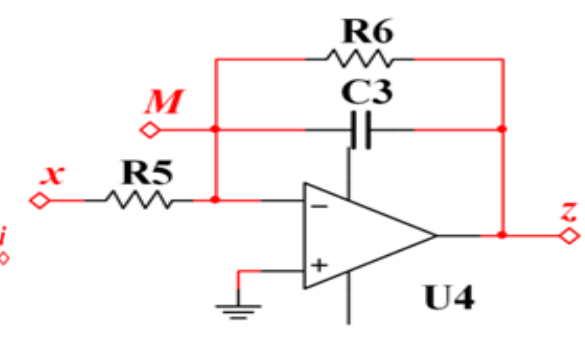

(b)

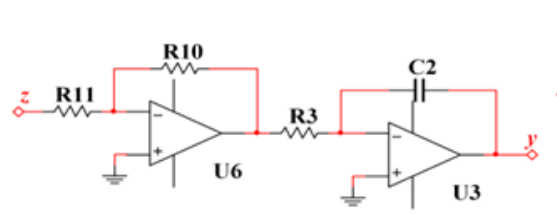

(c)

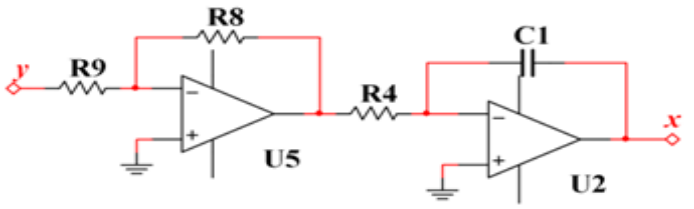

(d)

Fig.1 The analog circuit corresponding to the system, (b) (c) (d), memristor (a) 


\subsection{Memristor model description}

The HNN is described by the nonlinear differential equation of the group corresponding to $\mathrm{n}$ neurons [21]. In this paper, we consider the connection topology of a non-autonomous mHNN based on two neurons, which is shown in Fig.4. Here, $I_{m}$ and $F$ are amplitude and frequency parameters associated with the stimulus, by using the external stimulus input $I_{(s)}$ with a sine function $I_{(\mathrm{s})}=I_{m} \sin \left(2 p F_{\mathrm{s}}\right)$.

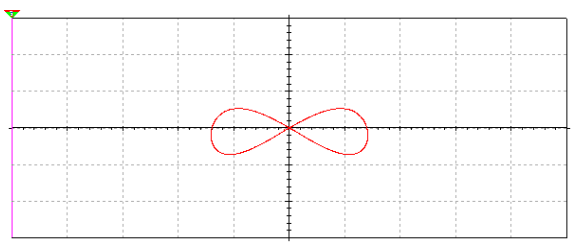

(a)

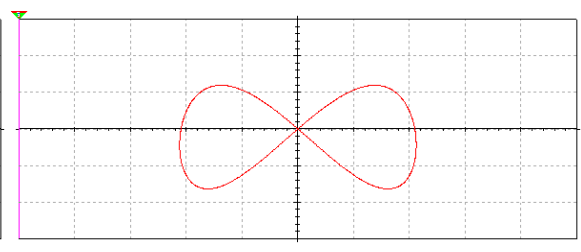

(b)

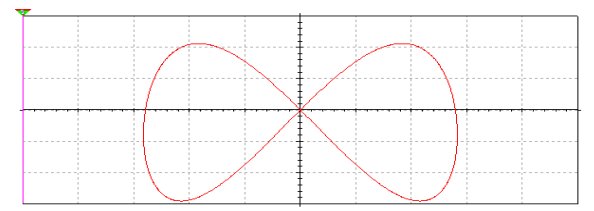

(c)

Fig.2 Numerical simulation of simplified pinch hysteresis loop of a memristor in the $v-i$ plane, (a) $f=1 \mathrm{kHz}$ with $v=2 \mathrm{~V}$ (b) $f=1 \mathrm{kHz}$ with $v=3 \mathrm{~V}$ (c) $f=1 \mathrm{kHz}$ with $v=4 \mathrm{~V}$

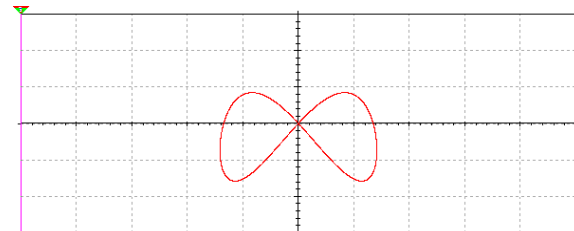

(a)

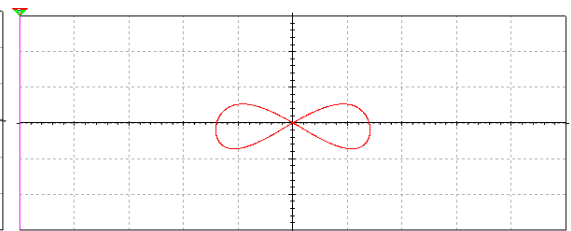

(b)

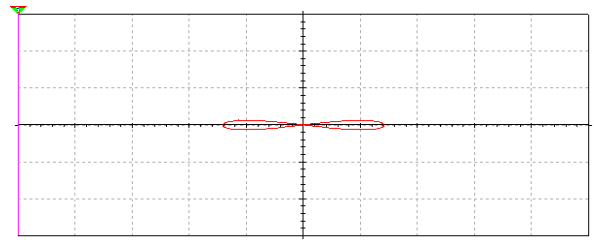

(c)

Fig.3 Numerical simulation of simplified pinch hysteresis loop of a memristor in the $v-i$ plane: (a) $f=500$ Hz with $v=2 \mathrm{~V}$ (b) $f=1 \mathrm{kHz}$ with $v=3 \mathrm{~V}$ (c) $f=5 \mathrm{kHz}$ with $v=4 \mathrm{~V}$ 
The model of memristor is obtained by Kirchhoff's law, and the basic relationship of discrete elements is expressed as follows

$$
\left\{\begin{array}{l}
i=W\left(v_{0}\right) v=\frac{1}{R} v_{0} v \\
\frac{d v_{0}}{d t}=-\frac{1}{R_{e}}\left(v_{0}+v\right)
\end{array},\right.
$$

The current and voltage are represented by $I$ and $V$ separately, $V_{0}$ is the internal state variable and $g$ is the gain of the multiplier $M_{1}$.

\subsection{Simplified memristor emulator-based jerk circuit}

In particular, resistors $R_{a}$ and $R_{b}$ are used as tunable circuit parameters are $R=10 \mathrm{k} \Omega, R_{a}=$ $80, R_{b}=5 \mathrm{k} \Omega, C_{1}=C_{2}=C_{3}=10 \mathrm{nF}$. In Fig. 1 , the voltage at both ends of the second order memristor diode bridge and the current flowing through the bridge are represented by $V$ and $I$, respectively. According to [13], the voltage-current relationship of the simplified memristor is expressed as

$$
\left\{\begin{array}{l}
C_{1} \frac{d v_{1}}{d t}=\frac{1}{R_{a}} v_{2} \\
C_{2} \frac{d v_{2}}{d t}=\frac{1}{R_{b}} v_{3} \\
C_{3} \frac{d v_{3}}{d t}=-\frac{1}{R_{c}} v_{1}-\frac{1}{R_{a}} v_{3}-\frac{g}{R} v_{0} v_{2} \\
C \frac{d v_{0}}{d t}=-\frac{1}{R_{e}}\left(v_{0}+v_{2}\right)
\end{array},\right.
$$

Where $V_{0}$ is the voltage of capacitor $C$ for the second-order memristor in Fig.1, the voltages $V_{0}$, $V_{1}, V_{2}$, and $V_{3}$ at both ends of capacitors $C_{1}, C_{2}, C_{3}$ and $C$ are treated as variables. Since the output voltage of $U_{4}$ is equal to $V_{2}$, the input voltage of the second-order memristor satisfies the relation $V_{2}=V$.

By introducing parameters and new variables in dimensionless form 


$$
\left\{\begin{array}{l}
x_{1}=\rho v_{1} \\
x_{2}=\rho v_{2} \\
x_{3}=\rho v_{3} \\
x_{4}=\rho v_{4} \\
\tau=\frac{t}{R_{c}}, C=C_{1}=C_{2}=C_{3} \\
a=\frac{1}{R_{a}}, b=\frac{1}{R_{b}}, c=\frac{1}{R_{c}}, g=\frac{g}{R}, e=\frac{1}{R_{e}}
\end{array}\right.
$$

The circuit equation in can be converted into a normalized system equation

$$
\left\{\begin{array}{l}
x_{1}=a x_{2} \\
x_{2}=b x_{3} \\
x_{3}=-c x_{1}-a x_{3}-g x_{4} x_{2} \\
x_{4}=-e\left(x_{4}+x_{2}\right)
\end{array} .\right.
$$

Set the following parameters: $a=12.3, b=2.15, c=4.4, e=4.0, g=1.2$.

\subsection{Eigenvalues and equilibrium point.}

That means $x_{1}=x_{2}=x_{3}=x_{4}=0$. It is easy to solve that a primary equilibrium point occurs at (4) which can be express as: $\mathrm{E}=(0,0,0,0)$.

The Jacobian [20] at $\mathrm{E}$ is derived as

$$
J=\left[\begin{array}{cccc}
0 & a & 0 & 0 \\
0 & 0 & b & 0 \\
-c & -g w & -a & g y \\
0 & -e & 0 & -e
\end{array}\right]
$$

This characteristic root equation is:

$$
\operatorname{det}(\lambda E-J)=0
$$

When this parameters are setting as $a=12.3, b=2.15, c=4.4, g=1.2, e=4$. And this eigenvalues corresponding to the equilibrium point are $\lambda_{1}=0.1547, \lambda_{2}=-0.9030, \lambda_{3}=-0.9030, \lambda_{4}=0.9030 \mathrm{i}$, There are three positive numbers $\lambda_{1}, \lambda_{2}$ and $\lambda_{3}$, and a conjugate complex numbers $\lambda_{4}$. So this system has a saddle-focus equilibrium point $(0,0,0,0)$. 


\section{Numerical diagram of the dynamical behaviors}

Two variable system parameters are determined by formula (3) as $b$ and $c$. The parameter region of interest is specified as $b \in[1.9,3], c \in[4,6]$.

\subsection{System phase diagram}

When this system parameters $a=12.3, b=2.15, c=4.4, e=4, g=1.2$, the time step is $0.01 \mathrm{~s}$, and the initial conditions are $(1,1,1,1)$, the four Lyapunov indices $L_{1}=9.7061, L_{2}=-0.0217$, $L_{3}=-3.3504$, and $L_{4}=-12.9289$, by calculation respectively under this parameter condition. Fig. 4 show the phase diagram of chaotic attractor obtained through simulation.

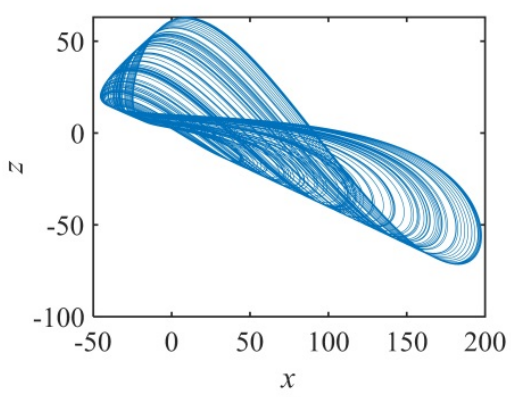

(a)

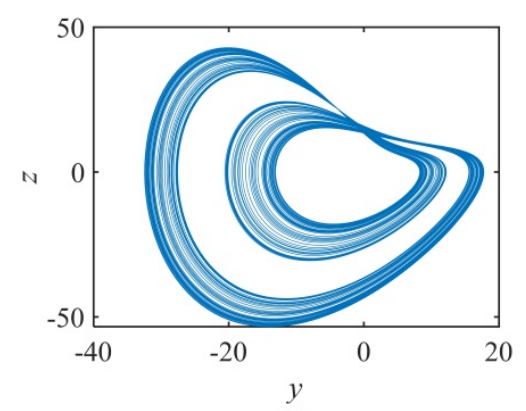

(b)

Fig.4 When $a=-12.3, b=-2.15, c=4.4, e=4, g=1.2$ chaotic attractor phase diagram, (a) $x-y$ plane (b) $y-z$ plane

\subsection{Lyapunov exponential spectrum and bifurcation diagram of the system}

This new system is a 4-dimensional, and its states under different parameters can be obtained by combining bifurcation diagram with Lyapunov exponential spectrum. In the following, parameters $b$ and $c$ are taken as variables, initial values are set as $(1,1,1,1)$, step size $h=0.01 \mathrm{~s}$, remaining parameters of the equation are fixed, and different states of the chaotic system are observed by changing parameters $b$ and $c$.

Take the parameter $b \in[1.9,3]$, and let $a=12.3, c=4.4, e=4.0, g=1.2$. Fig. 5 shows the Lyapunov exponential spectrum and bifurcation diagram of a chaotic system. According to Fig.5, when $b=2.3$, By finding the maximum Lyapunov exponent $L_{1}$ is equal to 0 , we know that the system is in a limit cycle state. When $b \in[2.6,2.75]$, When the maximum Lyapunov index $L_{1}$ is less than 0 , the system is in a periodic state.It can be seen from the figure that at other times, the maximum Lyapunov index $L_{1}$ is always greater than 0 is less than 0 , and the system presents a periodic state. At other times, the maximum Lyapunov exponent $L_{1}$ is 
always above the zero coordinate line , $L_{2}=0$, and other Lyapunov exponents are all less than 0 , so the system presents a chaotic state.

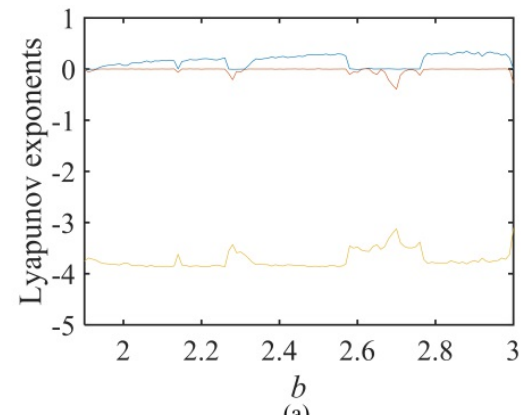

(a)

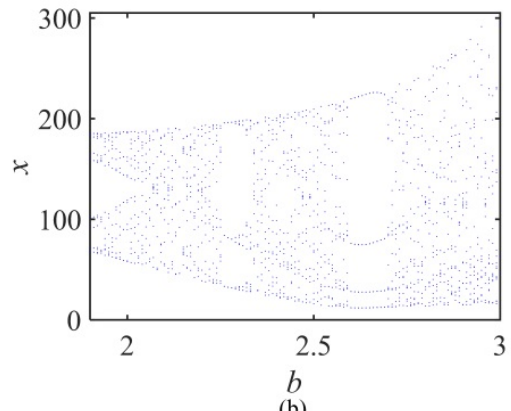

(b)

Fig.5 Lyapunov exponential spectrum and bifurcation diagram of this system when a change

Fig.6 shows this phase diagram of $x-z$ when the parameters $a=12.3, c=4.4, e=4, g=1.2$ are fixed, and $b=2.3$. When $b=2.3$, the phase diagram shows the limit cycle state. When $b=2.9$, the phase diagram is in a chaotic condition. In the meantime the Lyapunov exponent of this system is both less than 0 , one is equal to zero and the other is greater than 0 , which is in a chaotic condition. Through analysis, it can be easy seen that this result is integrally corresponding to Lyapunov exponential spectrum and the bifurcation diagram shown above.

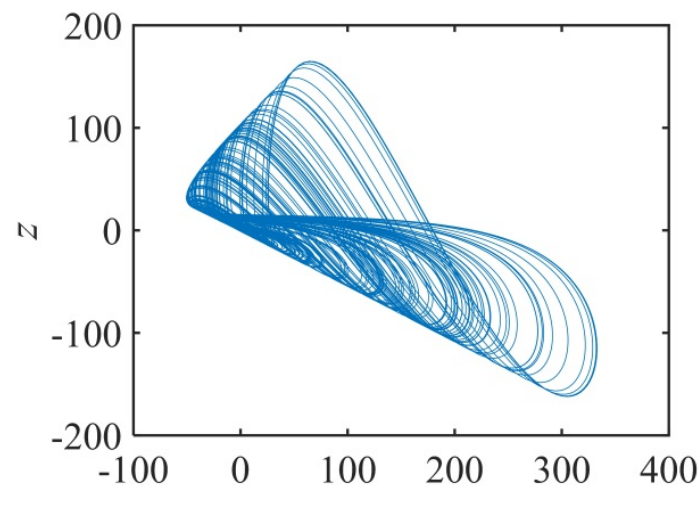

$x$
(a)

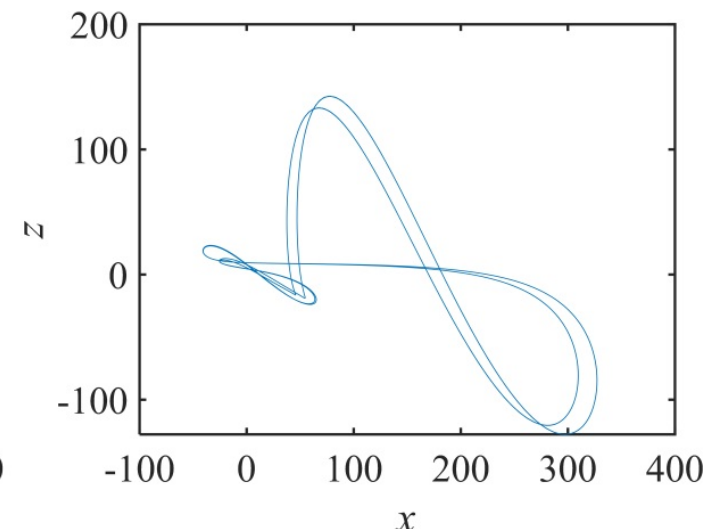

(b)

Fig.6 The phase diagram of the system is $b$ (a) $b=2.9$ (b) $b=2.3$

With the parameter $c \in[4,6], b=2.15, a=12.3, e=4, g=1.2$, Fig. 8 shows the Lyapunov exponential spectrum and the bifurcation diagram. When $c \in[4.65,4.73]$, the maximum Lyapunov exponent $L_{1}$ is equal to 0 , and the system appears as a limit cycle. When $c \in[4.74$, 5.26], the maximum Lyapunov exponent $L_{1}$ is greater than 0 , and no other exponent is greater than 0 , then the system shows a chaotic state. When $c \in[5.67,6]$, When the maximum 
Lyapunov index $L_{1}$ is greater than 0 , and the other indices are not greater than 0 , the system can be known to be in a chaotic state is greater than 0 , and no other exponent is greater than 0 , the system shows a chaotic state. When $c \in[5.29,5.68]$, When the maximum Lyapunov exponent $L_{1}$ is equal to 0 , then we know that the system is represented as a limit cycle.

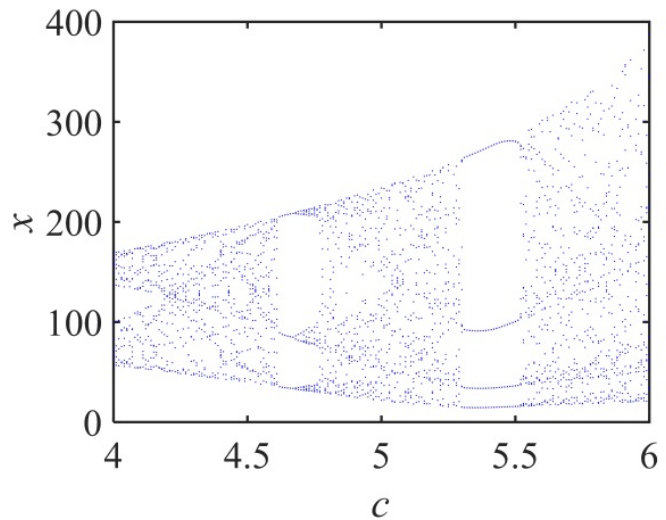

(a)

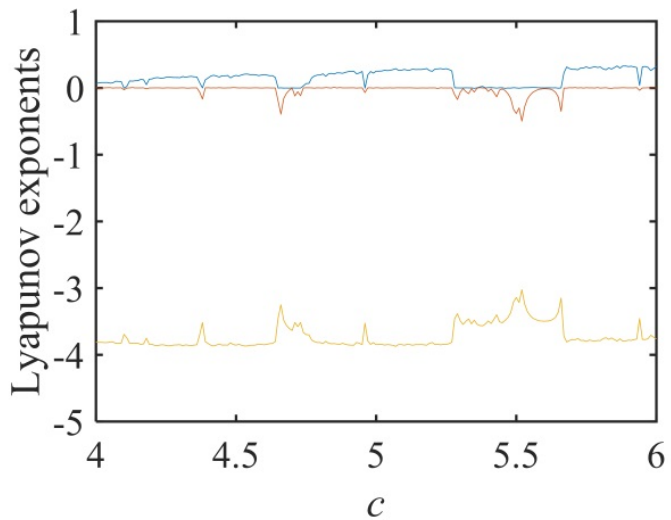

(b)

Fig.7 Bifurcation diagram of the system and Lyapunov exponential spectrum when a change (a)

Bifurcation diagram (b) Lyapunov exponential spectrum

Fig. 8 shows the phase diagram of $x-y$ when the parameters $b=2.15, a=12.3, e=4, g=1.2$ are fixed, and $c$ is $4.7,5,5.4$, and 5.9, respectively. When $c=4.7$ and $c=5.4$, the phase diagram is limit cycle. When $c=5$ and $c=5.9$, the phase diagram is in a chaotic condition. When the Lyapunov exponent of the system is less than 0 , one is equal to 0 , and the other is always above 0 , so we know that the system is in a chaotic state. Through the above analysis, it can be seen that this result is completely corresponding to the bifurcation diagram, and the Lyapunov exponential spectrum of the above system is in a chaotic state. Through analysis, it is easy to see that this result completely corresponds to the bifurcation diagram and Lyapunov exponential spectrum mentioned above. 

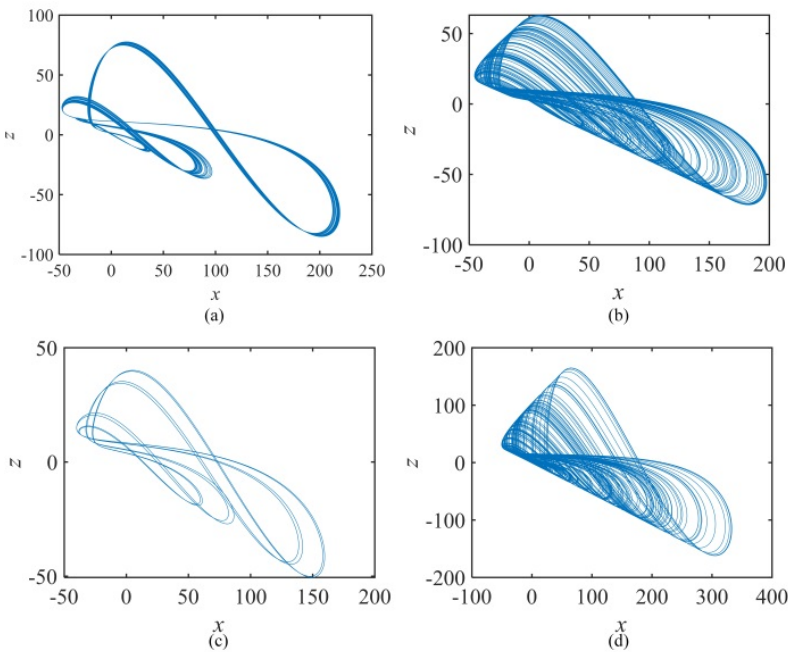

Fig.8 The phase diagram of the system is $c$ (a) $c=4.7$ (b) $c=5$ (c) $c=5.4$ (d) $c=5.9$

\subsection{The analysis of System complexity}

The complexity research involves scholars, and various fields in different fields have imparity understandings of complexity. Up to now, there is no qualitative concept of complexity. The complexity of the system can be known by the proximity between the chaotic sequence and the random sequence measured by the correlation algorithm. The higher the complexity value is, the higher the random sequence is and the higher the corresponding security is.

The analysis of system complexity includes complexity of behavior and structure. Behavioral complexity refers to the possibility of generating new models within a short time window measured by some method from the chaotic sequence itself. The more likely a new model is to be generated, the more complex the sequence becomes. Now, there are some algorithms to calculate the behavior complexity of the chaotic pseudo-random sequences, most of which are based on Shannon entropy and Kolmogorov method. These algorithms are quick in calculation and relatively accurate in results. However, if the dimension of pseudo-random sequences is too high or the symbol space of pseudo-random sequences is too large, the calculation results will overflow or even get no results. Structure complexity refers to the frequency characteristics through the transformation domain. The more balanced the energy spectrum distribution in the sequence transformation domain is, when the original sequence is closer to the random signal, the complexity of the sequence is greater. The corresponding spectral entropy value can be calculated by combining the concept of Shannon entropy. The 
energy characteristics of the transformation domain are analyzed by structural complexity, which is aimed at whole sequences, not just local sequences, so compared with the behavior complexity algorithm, the results have global statistical significance. In this paper, SE and C0 algorithm are used to analyze the structural complexity.

In this part, parameter $c$ is used as a variable to analyze the complexity of the system. When parameters $b=2.15, a=12.3, e=4, g=1.2$ are fixed, and $c \in[4,6]$, Figure 9 shows the simulation results.
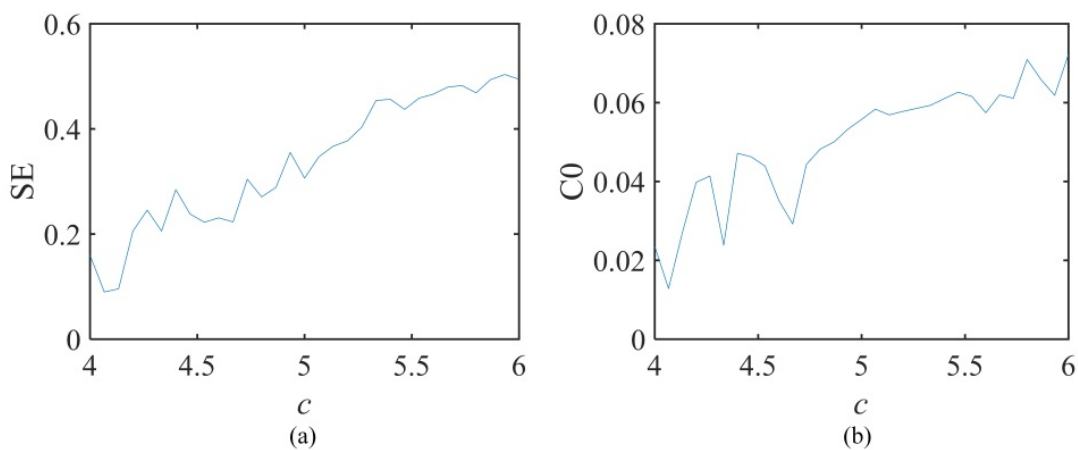

Fig.9 shows that $\mathrm{SE}$ algorithm and $\mathrm{C} 0$ algorithm have a high degree of synchronization

\section{Conclusion}

In the paper, we designed this new chaotic circuit and introduce its dimensionless equation. A new chaotic attractor is found in this chaotic system. By analyzing the Lyapunov exponential spectrum, bifurcation diagram and complexity, The dynamic nature was discovered, which show a high degree of complexity and sensitivity with the change of system parameters. Through numerical simulation, we observe the special phenomenon of chaotic. At the same time, some reasonable explanations are given for these phenomena. Because of these rich dynamic behaviors, this new chaotic system has a good application prospect in information encryption and secure communication.

\section{References}

[1] K. Eshraghian, O. Kavehei, K.R. Cho, J.M. Chappell, A. Iqbal, S.F. Al-Sarawi, D. Abbott, Proc. IEEE 100, 1991 (2012).

[2] B.C. Bao, T. Jiang, G.Y. Wang, P.P. Jin, H. Bao, M. Chen, Nonlinear Dyn. 89, 1157 (2017).

[3]. M. Chen, B.C. Bao, T. Jiang, H. Bao, Q. Xu, H.G. Wu, J. Wang, Int. J. Bifurc. Chaos 28, 1850120 (2018) 
[4]. M. Chen, M.X. Sun, H. Bao, Y.H. Hu, B.C. Bao, IEEE Trans. Ind. Electron. 67, 2197 (2019)

[5]. A.L. Fitch, D.S. Yu, H.H.C. Iu, V. Sreeram, Int. J. Bifurc. Chaos 22, 1250133 (2012)

1058 The European Physical Journal Special Topics

[6]. I. Petras, IEEE Trans. Circuits Syst. II: Express Briefs 57, 975 (2010)

[7]. S.P. Wen, Z.G. Zeng, T.W. Huang, Phys. Lett. A 376, 2775 (2012)

[8]. Q. Xu, Y. Lin, B.C. Bao, M. Chen, Chaos Soliton Fractals 83, 186 (2016)

[9]. H. Bao, W.B. Liu, M. Chen, Nonlinear Dyn. 96, 1879 (2019)

[10]. B.C. Bao, L. Xu, N. Wang, H. Bao, Q. Xu, M. Chen, AEÜ Int. J. Electron. Commun. 94, 26 (2018)

[11]. J. Kengne, Z.N. Tabekoueng, V.K. Tamba, A.N. Negou, Chaos 25, 103126 (2015)

[12]. Z.N. Tabekoueng, J. Kengne, H.B. Fotsin, A.N. Negou, D. Tchiotsop, Chaos Solitons Fractals 91, 180 (2016)

[13]. H.G. Wu, B.C. Bao, Z. Liu, Q. Xu, P. Jiang, Nonlinear Dyn. 83, 893 (2016)

[14]. J.H. Zhang, X.F. Liao, AEÜ Int. J. Electron. Commun. 75, 82 (2017)

[15]. V.T. Pham, S. Vaidyanathan, C.K. Volos, S. Jafari, N.V. Kuznetsov, T.M. Hoang, Eur. Phys. J. Special Topics 225, 127 (2016)

[16]. J.C. Sprott, IEEE Trans. Circuits Syst. II: Express Briefs 58, 240 (2011)

[17]. C.B. Li, J.C. Sprott, H.Y. Xing, Phys. Lett. A 380, 1172 (2016)

[18]. H. Bao, N. Wang, B.C. Bao, M. Chen, P.P. Jin, G.Y. Wang, Commun. Nonlinear Sci. Numer. Simul. 57,264 (2018)

[19].Xu Q, Lin Y, Bao BC, Chen M. Multiple attractors in a non-ideal active voltage-controlled memristor based Chua's circuit. Chaos Solit Fractals 2016;83:186-200.

[20].A. Wolf, J.B. Swift, H.L. Swinney, J.A. Vastano, Physica D 16, 285 (1985).

[21].Bao BC, Qian H, Xu Q, Chen M, Wang J, Yu YJ. Coexisting behaviors of asymmetric attractors in hyperbolic-type memristor based Hopfield neural network. Front Comput Neurosci 2017; 11:81.

[22].Chen, Chen; Sun, Kehui; He, Shaobo An improved image encryption algorithm with finite computing precision.107340 (2020). 